\title{
EL PROCESO DE DUELO DESDE LA TERAPIA FAMILIAR SISTÉMICA: EL PAPEL DEL APEGO ADULTO
}

\author{
ROCÍO GARCÍA TORRES ${ }^{1}$
}

Fecha de recepción: septiembre de 2018 Fecha de aceptación y versión definitiva: noviembre de 2018

RESUMEN El objetivo del trabajo que se propone es realizar una investigación en torno a la bibliografía existente sobre el proceso de duelo en el contexto de la terapia familiar sistémica, con el fin de estudiar qué peso tienen variables como el apego, el sistema de creencias familiar. No hay dos procesos de duelo iguales ni dos familias que vivan esta situación de la misma manera, por ello, a la hora de abordar terapéuticamente la pérdida de un ser querido, es necesario atender a sus diferencias culturales, a su sistema de creencias característico, y a las posibles disfunciones que pudieran preexistir.

PaLABRAS ClaVE: sistema familiar; duelo; vínculo; apego; pérdida.

\section{The experience of the process of grieving through systemic family therapy}

ABSTRACT: The aim of this paper is to conduct an investigation around the existing bibliography on the grieving process through systemic family therapy, in order to study the weight that variables such as attachment or the family's belief system. No two grieving processes are the same, nor two families experience this situation in the same way. Therefore, when it comes to therapeutically addressing the loss of a loved one, it is necessary to address their cultural differences, their characteristic belief system, and the possible dysfunctions that could pre-exist.

KEY WORDS: family system; grief; bond; attachment; loss.

1 Estudiante de doctorado en Universidad Pontificia Comillas. Correo electrónico: rogarcitoreres@hotmail.com. 


\section{LA MUERTE Y EL VÍNCULO: EL PROCESO DE DUELO}

La experiencia de los diferentes miembros de un sistema familiar ante la pérdida de otro integrante, es, probablemente, la tarea más difícil que pueda hacerse frente dentro del conjunto de vivencias que acontezcan en una familia; pero no es la misma tarea la que debe enfrentar dicha familia si la persona que fallece lo hace tras enfrentarse a una enfermedad que si esta muerte tiene lugar de forma repentina, súbita, como puede ocurrir en el caso de un accidente o de una catástrofe.

En el caso de tener que encarar una muerte repentina o una pérdida que con gran probabilidad tendrá lugar con inminencia, los recursos de los familiares son limitados en comparación con las demandas que la situación, sin duda difícil, requiere. En estos momentos es característica la toma de decisiones que la familia tiene que enfrentar y, sin duda, su situación anímica y emocional no estarán al servicio de estas labores, lo cual hace que la figura de los profesionales de la psicología sea de vital importancia en estos momentos. Los familiares de la persona fallecida o en estado crítico tendrán en estos momentos que afrontar situaciones que, probablemente, ni se habían imaginado con anterioridad y ello puede significar que la falta de entrenamiento o el no haberse planteado antes estas cuestiones suponga la no disposición de las herramientas necesarias para vencer la situación de forma sencilla (Espina, Gago y Pérez, 2005).

La diferencia principal en este sentido entre las distintas formas en las que se puede perder a un ser querido, hace referencia a la preparación psicológica que tienen los miembros de la familia con respecto a continuar viviendo sin esa persona que, hasta entonces, formaba parte de su día a día y de una forma trascendental. Tanto la muerte inminente como la pérdida anticipada de un miembro significativo, supone una gran desestructura para el sistema familiar. Tanto haber perdido a la persona en cuestión como saber que ésta se encuentra en fase terminal y su partida es inminente e inevitable, son cuestiones que invaden la afectividad de quienes le rodean, dominando así esta afectación e incertidumbre todo el ambiente familiar (Rolland, 2000).

Respecto a la duración del duelo, no se cuenta con una respuesta definitiva, ya que en realidad se hace alusión a una serie de etapas que deben ir cumpliéndose con éxito, llegando a poder pensar en la persona fallecida sin que ello conlleve los niveles iniciales de sufrimiento que en realidad eran disfuncionales, pudiendo así volver a dirigir las propias emociones y sentimientos hacia el resto de sus vidas y de otros seres queridos vivos (Dávalos, García, Gómez, Castillo, Suárez y Silva, 2008). 
Cuando nos referimos al término proceso de duelo estamos haciendo por tanto alusión a una experiencia que tiene un comienzo y un fin, no tratándose en realidad de un momento, situación o estado puntual (Tercero y Navarra, 2010). En los momentos descritos, la idea de la muerte, el dolor y la separación, invaden de esta manera el sistema familiar y sólo conceptos como la elaboración del duelo y el hecho de encontrar la manera de reanudar la vida normal, son centrales, pues todo girará en torno a estas cuestiones en la mente de quienes viven esta experiencia.

En el caso de la muerte inminente, cuando ésta aún no se ha producido, el primer aspecto debería ser trabajar el hecho de explorar si se ha llegado a comprender la inevitabilidad de la muerte, de si los distintos miembros han llegado a ser conscientes de que no hay posibilidad de reversión, de curación. La familia debe abandonar toda esperanza de supervivencia, toda fantasía de que su ser querido se cure; es así como los profesionales deben esperar la manifestación, idiosincrásica y completamente variable de persona a persona, del dolor que supone asumir la ruptura de este vínculo, la pérdida por definición. En estos casos, la preparación para la muerte es el trabajo central que se debe realizar, y aunque no se disponga de mucho tiempo para ello, siempre será más beneficioso disponer de un pequeño espacio para trabajar las distintas emociones que se estén dando al respecto y poder encontrar la manera de despedir a la persona, de soltar y poder desprenderse del vínculo, pudiendo separarse así del ser querido en cuestión. Este proceso, aunque súbito y rápido, puede ser más saludable que el hecho de no contar con la posibilidad de expresarlo y trabajar en ello (Espina, Gago y Pérez, 2005).

\section{LA REACCIÓN DE LOS MIEMBROS DEL SISTEMA FAMILIAR ANTE LA PRESENCIA DE UNA CRISIS}

Cuando una familia tiene que hacer frente a una pérdida, todo el sistema debe reestructurarse y volver a establecer un nivel de funcionamiento que, al contar con uno menos de sus miembros, no será por tanto la misma unidad familiar. Así la desestructura que sufre una familia ante la pérdida es de una magnitud tal que no sólo cada uno de sus miembros debe realizar un trabajo propio, personal e individual para colocar esta ausencia y ubicar el hueco que deja en su vida la persona que ha fallecido, sino que el sistema en su conjunto deberá trabajar en esta dirección.

Esta tarea, difícil en cualquiera de los escenarios a imaginar posibles, toma una dificultad distinta en medida de quién sea la persona que muere 
y cuál sea el subsistema al que por tanto pertenece. No es lo mismo perder a un abuelo o a uno de los progenitores incluso cuando ya se ha formado una nueva familia, que la elaboración del duelo si quien fallece es un hijo o un hermano, pues las funciones que desempeña cada miembro en la unidad familiar serán del todo distintas, suponiendo por tanto un trabajo de recolocación y reorganización diferente. De la misma manera, las circunstancias en las que la persona se va influyen de manera decisiva a la hora de encajar la pérdida y de cómo retomar una cierta normalidad posterior, pues no será igual perder a alguien tras padecer durante años una enfermedad crónica que perderlo por un accidente o una catástrofe puntual e imprevista (PérezCárdenas y Sebazco-Pernas, 2000).

\subsection{Cuestiones estructurales}

Las cuestiones estructurales que afectan a una familia tras la muerte de un familiar se refieren concretamente al lugar que ocupa en el sistema la persona que fallece y a cuáles eran por tanto los roles que desempeñaba y que ahora, quedan descubiertos. En este sentido, cuanto más importantes sean dichos roles y funciones, más profunda y potencialmente complicada será la recolocación de la familia que ahora ya no cuenta con esta persona tan central y cuyo papel era tan decisivo en el funcionamiento cotidiano.

Si es uno de los padres el que pierde la vida, la tasa de cambio es inevitablemente mayor, ya que el subsistema filial era dependiente de quien ahora ya no está; la función tanto materna como paterna son las que más desestructura producen en el funcionamiento y orden familiares ya que es de este subsistema parental de quien depende el consiguiente desempeño y funcionamiento del día a día en una familia.

Ellos son los portadores de afectividad, protección, seguridad y educación y son los encargados de poner los límites que para los hijos suponen un entorno seguro y protegido en el que poder crecer e ir evolucionando como seres humanos, aprendiendo de ellos, de su mano, que es lo que deben y no deben hacer; aprendiendo en primer lugar de su ejemplo y recibiendo reforzamiento, tanto positivo como negativo en relación a la conducta que van desempeñando de forma cotidiana. Los padres son, en resumen, los que posibilitan que los pequeños del sistema familiar se desarrollen de forma satisfactoria y segura, cumpliendo los distintos hitos evolutivos que, acorde a su edad, les toque ir viviendo (Rolland, 2000).

En cambio, cuando una familia se enfrenta a la pérdida de un hijo, aunque el desajuste emocional puede llegar a ser exponencialmente mayor, la 
desestructura o el desajuste estructural no lo son tanto. De ellos no depende el orden, las normas, ni los ingresos económicos para sustentar un hogar, entre otras cuestiones de importancia trascendental. Aquí cabe señalarse que la culpa es un componente adicional que está muy presente en los casos de duelo por la muerte de un hijo, ya que la sensación de haberle fallado, desamparado o de haber fracasado como padres es un sentimiento frecuente en estos casos (Roitman, Armus y Swarc, 2002).

En este mismo aspecto, que el fallecido sea un hombre o una mujer también entraña una gran variedad de diferencias. Desde un punto de vista cultural, es comúnmente entendido que a un hombre le corresponden funciones de tipo más ejecutivo, como podría ser el hecho de dirigir una empresa familiar, por ejemplo. A las mujeres por su parte, le han sido asignadas funciones más relacionadas con el servicio y el cuidado, como podría ser la preocupación que ostentan por la correcta alimentación de los propios hijos o el cuidado de un enfermo. Esta idea culturalmente aceptada, de manera general, se asocia con la función materna de la mujer, más nutricia y emocional, y el hecho de repartir las funciones fuera de estas mencionadas pautas culturales suele conllevar una problemática implícita que requiere a su vez una gran labor de reestructuración y negociación explicita. De ahí que se den importantes diferencias en función de quién sea el miembro de la familia que desaparezca y de cuáles fueran sus labores o papeles desempeñados dentro del sistema al que pertenecía (Rolland, 2000).

De la misma manera, también existen diferencias en la vivencia del duelo en función del género, y se ha señalado que las mujeres dolientes tienden a presentar una sintomatología más depresiva, mayores niveles de estrés psicológico, presentando de esta manera un duelo más intenso, doloroso y duradero que el que podrían vivenciar los hombres, caracterizándose ellos por la expresión de su frustración a través de la ira en mayor medida (Volker, Striegel, Dudley, Wilkins y Gibson, 1997).

En el caso adicional de que antes de la muerte, tras un accidente, por ejemplo, la persona afectada quede gravemente impedida requiriendo los cuidados de uno de los miembros de su familia, esto entrañará una gran restructuración dentro de la misma, siendo el cuidador primario al que se haya elegido, el que va a contar con una mayor carga emocional, así como un mayor desgaste, tanto físico como psicológico. De esta manera es necesario tener en cuenta los factores personales, ambientales y situacionales que podrían llevar a dicho cuidador a sentir impotencia por el papel que debe enfrentar (Davidhizar, 1992).

En estos casos en los que la muerte se demora, siendo precedida de un tiempo de incapacidad o enfermedad grave, tanto el mencionado cuidador 
primario como el enfermo, deberán trabajar las renuncias a las que hacen frente al tener que vivir esa situación, así como los miedos presentes y futuros que deben abordar (Feigin, 1994). Cuando es a uno de los hijos al que le corresponde ostentar el puesto de cuidador primario, esto supone una complicación adicional, ya que esto podría implicar un retraso de su propia emancipación, así como una renuncia al hecho de vivir ciertos hitos evolutivos. Todo ello debe ser tenido en cuenta, así como trabajado terapéuticamente.

Es un punto al que es relevante otorgarle un especial lugar ya que el agotamiento que sufre la persona encargada de los cuidados, en ciertos casos llega a ser tal que las relaciones familiares se resienten y el vínculo incluso con el mismo enfermo se puede resentir. De ahí la importancia de apreciar la labor que hace quien renuncia a otros proyectos personales y a su tiempo, así como la importancia que tiene el hecho de contar con un espacio en el que poder tomar cierta distancia de descanso para volver a la labor de cuidado con la energía suficiente y así hacerlo con la capacidad que ello demanda.

\subsection{Cuestiones AFEctivas}

Enfrentar la pérdida de un ser querido siempre supone una tarea ardua y difícil, además de suponer un proceso largo que va a requerir una gran cantidad de trabajo personal para elaborarla correctamente y así poder retomar una cierta normalidad posteriormente, a pesar de que es necesario puntualizar que la normalidad previa a la pérdida nunca podrá volver a restaurarse. En este aspecto emocional y afectivo en el que el ánimo se ve tan resentido, es de gran importancia tener en cuenta una serie de aspectos para poder ir haciendo frente a este trabajo de superación de manera exitosa. En primer lugar, más allá de los muros que guardan la familia, es importante tener una amplia red social sobre la que poder apoyarse y con la que poder compartir los momentos más amargos que esta difícil situación conlleva (Millán-González y Solano-Medina, 2010).

En ocasiones, salir del ambiente familiar y respirar un aire distinto al que impera en el ambiente de la familia en cuestión, puede ser saludable, no sólo por lo que esto supone a efectos de la necesaria sociabilidad y del ocio, sino porque compartir momentos con quien está fuera del sistema, podría ser sin duda saludable para tomar distancia y, por tanto perspectiva, del círculo de tristeza que impera en una familia en la cual todos sin excepción están enfrentando la misma tarea y momento de reestructuración (Rolland, 2000). 
Esta red social cumple propósitos distintos, tales como recibir aprobación y apoyo con respecto al momento que se está atravesando y también ayuda a confirmar que ciertas creencias, sentimientos y puntos de vista son apropiados. Por tanto, se crea un sistema de apoyo que es decisivo para ir salvando los distintos obstáculos que entraña un duelo, para hacerlo acompañado, contando con el apoyo de personas significativas que, además, están en disposición de ofrecer dicha ayuda; aquí es importante recordar que los miembros de la familia de la cual se forma parte, están igual de heridos y difícilmente podrán dedicar sus energías a animar a otro. Esta red social de amigos y conocidos cercanos pueden ayudar enormemente al doliente no sólo a facilitar la expresión sincera y abierta de los sentimientos que le acechen en este difícil trance, sino que pueden ser fuente de ayuda tanto material como de información (Millán-González y Solano-Medina, 2010).

Es interesante mencionar que, aunque parece algo únicamente positivo, el apoyo social para paliar las cuestiones emocionales, puede resultar negativo cuando quien lo recibe se siente humillado al sentirse por debajo de quien ofrece la ayuda; de la misma manera, podría ser negativo o contraproducente para el doliente si dicho contacto supusiera que éste se sintiera más incapacitado y hundido. Esto ocurre cuando las relaciones no son igualitarias, ni recíprocas, o cuando está presente la ambivalencia de sentimiento (Rolland, 2000). En cualquier caso, el apoyo social parece ser un factor protector, tanto en situaciones de duelo como en situaciones de enfermedad.

\subsection{PERIOdos CENTRÍPETOS Y CENTRÍfugos EN EL CICLO DE LA VIDA FAMILIAR}

Los conceptos centrípeto y centrífugo en la terapia familiar sistémica hacen referencia, en definitiva, a la cohesión más alta, en el primer caso o más baja, como es el segundo caso. De esta manera, cuando una familia se encuentra más unida, más cohesionada entre sí, entre sus miembros, nos referimos a que está viviendo una etapa centrípeta, en la cual pareciera que sus miembros fuesen una unidad, estando más cohesionados o unidos que en otros momentos. En el proceso de duelo, estos conceptos tienen gran importancia ya que resultan relevantes para comprender el momento que atraviesa la familia que debe afrontar tan doloroso proceso y, en consecuencia, elaborar el plan de intervención específico, atendiendo a las peculiaridades propias de cada momento.

En casos tales como el periodo de nacimiento de los propios hijos o de su adolescencia, por ejemplo, las demandas de la situación requieren que se dé una mayor cohesión o unión porque así lo demanda la situación que se tiene 
que enfrentar, como es el caso de la recién llegada al mundo de los hijos o la adolescencia de los mismos, momento en el cual las tareas ponen el énfasis en la identidad personal y en la autonomía (Rolland, 2000).

Un periodo centrípeto significaría que hay una mayor unión o cohesión dentro del sistema en su conjunto y de sus miembros a nivel individual, entre ellos. Los momentos centrífugos conllevan que las energías se destinan a las tareas relacionadas con lo extra familiar, volcándose cada miembro en aspectos que tienen más relación con lo externo.

Es necesario tener en cuenta este aspecto ya que si quien fallece es un adolescente, encontrándose la familia por tanto en un periodo centrípeto, probablemente se tienda a una mayor cohesión entre sus miembros, lo cual es necesario observar con cautela, ya que no pueden dejarse de lado las demás tareas individuales que deben llevarse a cabo para seguir un desarrollo funcional con la correspondiente evolución a lo largo del ciclo de vida familiar.

Cuando una enfermedad o un fallecimiento conllevan un momento centrípeto, de una mayor cohesión entre sus miembros y, pasado un tiempo se vuelve a retomar la propia autonomía, la persona que se ha visto atrapada de alguna manera en el sistema familiar, en detrimento de su funcional evolución autónoma, puede querer retomar las tareas evolutivas que tenía por delante en el momento en el que ocurrió la crisis, y esto no siempre es algo realista o saludable.

\section{DIFERENCIAS CULTURALES EN LA EXPERIENCIA DE DUE- LO DENTRO DEL SISTEMA FAMILIAR}

Las adscripciones de raza, etnia y religión tienen gran peso e influencia en las creencias que cada familia tiene sobre la salud y la enfermedad (Zborowski, 1969). Es interesante destacar que en muchas ocasiones dichas creencias sobre la salud no emergen en la familia hasta que ésta precisamente tiene que hacer frente a una crisis relacionada con la salud de alguno de sus miembros.

A este respecto, cada familia tiene sus propias concepciones sobre cuál es el adecuado rol que el enfermo debe tener como paciente, que ya es de hecho, sobre quién debe ser el cuidador principal de la persona que está en estado crítico, sobre cuál debe ser la reacción ante el fallecimiento o sobre cuáles son los rituales a desarrollar o las expresiones y manifestaciones emocionales y afectivas permitidas o esperables tras el fallecimiento del ser querido (Yoffe, 2003). 
Este aspecto es de crucial importancia para los profesionales que estarán en contacto con dichas familias ya que las manifestaciones descritas variarán en función de quién sea la familia a la que se tenga que ayudar. Por ejemplo, los judíos o italianos, describen con mayor viveza y literalidad sus dolencias y síntomas, mientras que las personas procedentes de países anglosajones, suelen por el contrario minimizar, ocultar o disimular sus afecciones y dolencias (Rolland, 2000). Esto es importante tenerlo en cuenta para establecer una adecuada alianza terapéutica con los familiares a los que vayamos a atender, pues el hecho de agobiar y no respetar la distancia que unos pueden necesitar, podría hacer que se sintiesen asfixiados y necesitasen, por tanto, alejarse de nosotros, renunciando así a la ayuda beneficiosa que pudiéramos ofrecerles.

Por otro lado, si una persona en situación de crisis como es el hecho de hacer frente a la pérdida de un ser querido, necesita manifestar sus vivencias emocionales sin contención, con visible externalidad, debemos aprender a adaptarnos a ese tipo de manifestación del dolor para saber recogerlo y prestarle el apoyo que, en ese caso concreto, es el más beneficioso para la persona que tenemos delante, la única que puede ayudarle dadas sus demandas y características personales, tanto psicológicas como culturales. En este punto, es necesario dejar claro que el profesional al frente de la situación debe dejar de lado su propia concepción del dolor y de su manifestación, sus propias necesidades y vivencias así como su opinión sobre la familia que se tiene delante; de otra forma, dicho sistema familiar, el cual está vivenciando un momento de crisis y desestructura, no se sentirá apoyado y ayudado, sintiendo por el contrario que prefiere prescindir de nuestra ayuda y servicio, lo cual es un fracaso para nosotros como profesionales (Weiss, 1999).

Para concluir, puede puntualizarse en este punto que abandonar el deseo de controlar la situación conforme a lo que nuestras propias expectativas, manuales o experiencias nos guíen, es la mejor de las opciones, ya que adaptarnos al ritmo y necesidades de quienes tengamos delante serán las únicas opciones posibles para afrontar con éxito la situación, pudiendo ser una ayuda beneficiosa para aquellos a los que tengamos oportunidad de apoyar.

Respecto a los rituales, podemos señalar que tienen una importante labor en el funcionamiento psíquico de las personas, y estos también son muy variables en su forma y en su fondo en función de la cultura de la que provengan. Dichos procedimientos o rituales proporcionan a las familias que los ponen en marcha, estabilidad y continuidad en el tiempo y pueden ser realmente terapéuticos y beneficiosos para quien los practique, independientemente de la cultura de la que provenga. Se ha observado, que dentro de las familias tienen lugar cuatro tipos de rituales distintos pero que, a su vez, son 
ciertamente universales y comunes a todos los sistemas familiares, independientemente de su proveniencia o lugar de origen (Wollin y Bennet, 1984):

1. Rutinas cotidianas o interacciones familiares pautadas y practicadas de forma regular, como pudieran ser los encuentros a las horas de las comidas, los que tienen lugar antes de irse a dormir, los planes y actividades de ocio y fin de semana, etc.

2. Tradiciones familiares tales como celebraciones de cumpleaños, de aniversarios, etc. Este tipo de rituales fortalecen los lazos familiares y transmiten la identidad familiar de generación en generación, ya que son específicos e idiosincrásicos de cada sistema familiar.

3. Festejos, los cuales vinculan a la familia con una identidad cultural más amplia, con ideas y símbolos que comparten con su cultura, sociedad o religión. De este tipo serían la celebración de fiestas regionales o tradicionales propias de cada localidad, la navidad, la semana santa, el ramadán o el día de acción de gracias, entre otros.

4. Rituales del ciclo de vida, los cuales se refieren al paso de cada una de las etapas que nos toca ir viviendo, como pudieran ser los cumpleaños, los aniversarios, las graduaciones, las bodas o los funerales. Dentro de este grupo, que es a la vez el que más nos ocupa, también se encontrarían rituales inesperados que pudieran acontecer, como una enfermedad o situación de discapacidad ocurrido tras un accidente o situación de catástrofe.

A través de los rituales y de la forma misma que una familia tiene de enfrentarlos, los profesionales pueden observar gran cantidad de información sobre las dinámicas que imperan dentro de dicho sistema, así como de la vivencia que tienen de los sentimientos que les toque enfrentar. En este sentido, toda aquella familia que tenga dificultades para expresar sus sentimientos o permitirse un cierto nivel de afectividad, puede manifestar una actitud fría ante la vivencia y desarrollo de un ritual, no queriendo incluso participar en él. Esto ocurre ante patrones rígidos e inflexibles imperantes en las dinámicas familiares y conviene atenderlo y registrarlo ya que puede ser ciertamente patológico con posterioridad.

\section{EL PESO DEL SISTEMA DE CREENCIAS DE LA FAMILIA EN EL PROCESO DE DUELO}

Toda familia tiene un conjunto de normas, tanto implícitas como explícitas, que facilitan la interacción de sus miembros ya que funcionan como 
prescripciones acerca de lo que corresponde a cada rol, refiriéndose a las actitudes y acciones que lo deben caracterizar, así como respecto a las consecuencias de dichas acciones. Las normas de cada familia son idiosincrásicas, personales y en ningún caso, éstas son correctas o incorrectas (Walsh, 1993), pero en ocasiones, cuando dicha normativa es muy rígida o inflexible, si podría resultar disfuncional para el normal desarrollo de dicho sistema familiar en cuestión, ya que esto podría suponer que las relaciones entre sus miembros no estuvieran favoreciendo la deseable autonomía que es necesario establecer para el favorecimiento de la propia integridad así como de una adecuada salud mental, tanto a nivel individual para cada miembro, como a nivel grupal para el sistema familiar en su conjunto.

También es interesante destacar que la manera de afrontar las crisis en una familia, es un hecho que se puede conocer y que, como profesionales que vamos a trabajar con uno de sus miembros o a todos en conjunto ante otra situación de crisis, puede resultarnos útil. En este sentido, la forma de afrontar una situación estresante, el hecho de reaccionar al estrés mismo, son reacciones que pueden de alguna manera aprenderse, por ejemplo, por observación, dentro de un sistema familiar del que se forma parte. De esta manera, por ejemplo, una madre que cuando era niña, tuvo que hacer frente a según qué adversidades, como pudieran ser la muerte de uno de sus padres o incluso la separación de los mismos, seguramente se haya hecho con las herramientas suficientes para estar preparada a la hora de hacer frente a ciertas experiencias que supongan un elevado nivel de estrés (Espina, Gago y Pérez, 2005).

Las fortalezas se acumulan como si fueran herramientas que se llevan en una mochila a la espalda y, a la hora de tener que lidiar con otras situaciones que conlleven, estrés o sufrimiento de niveles similares, se podría estar más preparado para enfrentarse a ellas cuando se cuenta con herramientas que han sido útiles a la hora de lidiar situaciones similares en el pasado. En este sentido, todos desarrollamos en el núcleo de la familia de la que provenimos, un sistema de creencias, una filosofía personal que nos define y ubica a la hora de tener que enfrentar los desafíos comunes de nuestra vida (Kluckhohn, 1958); este sistema de creencias funciona como un mapa cognoscitivo que nos orientaría en la acción.

Sin duda, dicho mapa también se pone en funcionamiento cuando hay que encarar la muerte de un ser querido, sea un hecho o un acontecimiento inminente; se trata de un conjunto de creencias respecto a la forma de encarar la salud, la enfermedad y la muerte, que nos lleva a entender y encajar ésta última de una manera concreta, la cual hemos aprendido de alguna forma en el cultivo del ambiente familiar del que formamos parte. Aunque 
las creencias que cada miembro de la familia tenga puedan ser distintas en su forma y apariencia, el sistema familiar comparte de forma general un conjunto de valores significativos para todos los miembros que la integran.

Por su parte, Kleinman (1988) ya destacó la importancia de contar con un sistema de creencias, significados o modelos explicativos que dieran respuesta a ciertas cuestiones que pudieran desestabilizar a las personas ante situaciones de crisis o enfermedad, así como facilitar su adaptación a la misma. De esta manera, distinguió tres niveles de significado: la dimensión biológica de la enfermedad, la simbólica y la social, haciendo así una clara distinción entre los tres conocidos términos disease, illness y sickness.

Según esta clasificación, el primer de los apartados se referiría únicamente a un componente biomédico, biológico, el cual entiende nuestro cuerpo como una máquina que, por supuesto, se estropea y puede funcionar erróneamente al enfermar. La segunda concepción hace alusión a la propia experiencia que vive quien padece los síntomas y signos de la enfermedad, encontrando en esta concepción simbólica tanto al enfermo como a su familia, pues representa la manera en que el ser humano enferma. La última se refiere a los significados que han quedado asociados a un determinado padecimiento como resultado de fuerzas mayores, de tipo macrosocial, económico, político, etc. como pudieran ser la pobreza, la exposición a la contaminación, un concreto estilo de vida indeseable o la alimentación (Sontag, 1978).

Es importante hacer mención a todos estos componentes de una enfermedad ya que el sistema de creencias que impera en una familia funcionan como mediadores o traductores entre la propia experiencia de enfermedad y muerte y la experiencia personal que tiene la familia. Por otro lado, cuando una familia debe hacer frente a la posibilidad inminente de pérdida, el hecho de elaborar un significado para la enfermedad que mantenga el propio sentido de capacidad, es una tarea de vital importancia en el seno familiar: contar con la sensación de poder controlar la situación, de creer que los propios recursos están a la altura de las demandas de la misma (Espina, Gago, y Pérez, 2005).

En este sentido, el sistema de creencias es fundamental con respecto a qué y quién tienen el poder de influir en el curso de los acontecimientos que se vayan desarrollando. Esto es especialmente delicado cuando uno o varios de los miembros de la unidad familiar se puedan sentir culpables por el hecho que ha llevado a la persona en estado crítico o fallecida a su situación. Si aún no ha muerto, pero se encuentra en estado grave, cualquier hecho que suponga un empeoramiento de su estado, podría llevar a un completo desmorone familiar dando lugar a una crisis dentro de la familia en sí (Grinberg, 1963). 
Esto tiene que ver con la ambigüedad que acompaña al hecho de no saber cuáles son los factores que pueden causar un determinado accidente o enfermedad, nos estamos refiriendo a factores psicosociales que hacen difícil identificar dentro de una familia, cuáles son los comportamientos o actitudes que pudieran llevar a un desenlace trágico; materializándose esto en errores de actuación vividos con culpa por los familiares, así como omisiones detectadas por los mismos. En estas situaciones en las que se da una amenaza de pérdida, es importante saber que las mujeres están más expuestas a sentir culpa o vergüenza, pues existen expectativas al respecto que las sitúan cultural y socialmente, como el principal cuidador, tanto de sus hijos, como de su marido, como de su padre anciano o su familia extensa (Rolland, 2004).

El sistema de creencias aquí es realmente importante ya que el hecho de colocarse a uno mismo como excesivo responsable, mientras se deja de lado el inevitable peso que tiene la suerte, el azar o sencillamente todo lo que es ajeno a nuestro control, puede suponer un grave problema en aquel que se coloca como responsable de lo ocurrido, tanto por haber cometido algún tipo de error a su criterio, como por no haber hecho algo concreto que dicha persona creía necesario hacer para evitar la catástrofe en cuestión.

En este sentido, un excesivo locus de control interno podría no ser un factor protector ya que supondría para la persona portadora de dicho constructo una increíble fuente de sufrimiento y culpabilidad. No todo está en nuestra mano ni somos todopoderosos, a veces no nos corresponde el poder de obrar una salvación o de evitar el desastre.

\section{LA VIVENCIA DE LA FAMILIA ANTE LA FASE TERMINAL DE UN MIEMBRO}

Cuando tiene lugar una catástrofe en la cual el miembro de una familia no fallece en el momento, sino que se queda gravemente impedido o herido después de su ocurrencia, dicho sistema familiar va a tener que hacer frente a un proceso duro y doloroso en el cual el punto central será prepararse para despedir a dicha persona.

Esta última fase se conoce con el nombre de fase terminal y en ella la característica más definitoria es la inevitabilidad de la muerte; este camino irreversible es palpable en la familia, pues este hecho pasará a dominar por completo la vida familiar. Durante esta fase tienen lugar tres periodos: el duelo, la congoja y la elaboración de la pérdida (Rolland, 2004). Y son 
centrales en este momento cuestiones tales como la separación, la pérdida misma, la despedida, el dolor y el planteamiento de cómo reanudar la normalidad y el funcionamiento previo de cada miembro de la familia en cuestión.

Los profesionales de la psicología que vayan a tratar con los familiares que estén atravesando este momento difícil deben saber y ser plenamente conscientes de que su actuación empieza cuando las personas que integran esta familia manifiestan el duro dolor que se desprende de haber asumido que la situación es irreversible. Por ello, lo central en este momento es una actuación siempre encaminada a apoyar a los familiares que, en este primer término, deben abandonar toda esperanza y ser conscientes de que la muerte del ser querido que nos ocupa, es un hecho sin opción a cambio. Dicha manifestación de dolor sólo se da cuando los familiares abandonan la batalla por mantener con vida a su ser querido, batalla en la que han podido verse inmersos en un primer momento con facilidad (Toro et al, 2007).

Cabe mencionarse que es una etapa especialmente delicada, pues los familiares deberán hacer frente a una serie de tareas nada sencillas, pero realmente inevitables, tales como: quién cubrirá las funciones prácticas del ser que va a irse con inminencia o la posibilidad de dejar bien estructurado todo lo relativo al testamento del enfermo, así como la posibilidad misma de redactar el documento, en caso de ser posible. Como puede intuirse, en estos casos en los cuales la muerte es un hecho inminente, pero habiéndose dado éste de forma repentina y súbita, la dificultad y dureza de la situación se agrava, pues no ha habido una reparación previa en la cual los familiares se hayan podido ir reconciliando con la idea de perder a ese ser querido, como pudiera ocurrir en los casos de fallecimiento tras una larga enfermedad.

Por todas estas cuestiones, la presencia y el apoyo de un preparado conjunto de profesionales de la psicología tiene gran importancia, pues el acompañamiento a estas personas puede resultar en primera instancia, útil y práctico; radicando aquí el interés principal del presente artículo: llevar a cabo un abordaje adecuado y personalizado en la intervención con personas que atraviesan un proceso de duelo ya que, como señalábamos anteriormente, los profesionales de la psicología no deberán nunca inducir un determinado estado ni forzar el ritmo de los dolientes para que se sitúen en la etapa más deseada con respecto a los tiempos y etapas que van aconteciéndose para el enfermo.

Nuestra actitud deberá ser en todo momento prudente y al servicio de las necesidades de la persona a quien debamos ayudar. La primordial tarea es ser un apoyo para los familiares; ofrecerles nuestra ayuda para dar con la información que necesiten o acompañarles en las duras tareas que deban 
enfrentar, son en todo momento los objetivos principales de nuestro trabajo con ellos (Gil-Juliá, Bellver y Ballester, 2008).

Otra cuestión realmente delicada de esta etapa es el hecho de decidir si mantener al familiar enfermo con vida, cuando esta cuestión es planteada a la familia por parte del equipo médico. Puede que los familiares prefieran no verle sufrir y decidan dar un descanso a dicha persona, pero a la vez puede que prefieran alargar su vida el máximo tiempo posible, pudiendo conllevar ambas decisiones un sentimiento de culpabilidad intenso o de duda trascendental, tales que supongan una fuerte carga para los familiares que toman la decisión. Tanto acortar la agonía de alguien a quien se quiere como querer alargar su vida todo lo que en sus manos quede, puede llevarles a sentir que su decisión ha nacido de un deseo personal y propio, y éste es un aspecto de gran importancia a tener en cuenta a la hora de trabajar con estas familias. Al duro sentimiento de tristeza se suman otras cuestiones afectivas, morales y éticas que hacen del duelo anticipado un entramado de complejo abordaje, el cual debemos trabajar con extrema cautela y prudencia (Toro, Flores, Gil, Domínguez, Marín y Prado, 2007).

También puede darse la circunstancia de tener que otorgar un poder notarial a algún miembro del sistema familiar si el paciente ha perdido determinadas capacidades. Es momento de tener en consideración, o no hacerlo, los deseos manifiestos del enfermo con respecto a cómo encarar su muerte; si deseaba (y fuera posible) donar órganos, si deseaba un entierro o una incineración, si prefiere incluso morir en casa o en el hospital (cuando la elección sea posible). En estos duros momentos, puede que también sea preciso tomar este tipo de decisiones y enfrentar ciertas burocracias en las cuales nadie desea verse sumido dada la situación de máxima tristeza; también un profesional de la psicología podría ofrecer su ayuda en la medida en que pudiera ser de utilidad a los familiares en el desempeño de estas cuestiones o tareas (Cobos, Espinoza, Portillo y Rodríguez, 2002).

Por tanto, nuestra labor como psicólogos, lejos de tener una intención psicoterapéutica en un primer momento con la que ayudar a los familiares a que trabajen y elaboren de forma profunda y sistematizada su duelo y su dolor, consistirá precisamente en acompañar y apoyar a todo aquel que lo necesite y desee, estando al servicio de sus necesidades. No olvidemos que estamos allí para facilitarles este duro momento y serán ellos quienes, de alguna manera, nos orienten en cómo desempeñar nuestra labor facilitadora. Si ellos lo desean y tenemos posibilidad, en un futuro les ofreceremos nuestros servicios para continuar con la elaboración del duelo de una forma más prolongada en el tiempo, con la ayuda de una buena psicoterapia y de sus correspondientes herramientas. Tener en cuenta todas estas consideraciones 
parece el punto de mayor relevancia a la hora de hacer un adecuado acompañamiento en el proceso de duelo dentro del contexto familiar.

\subsection{DiSFUnCIÓN FAMILIAR PREEXISTENTE}

Aquellas familias que presentan problemas encapsulados o latentes deben ser diferenciadas de aquellas que cuentan con pautas disfuncionales, las cuales son más generalizadas en sus diferentes campos de desempeño, además de arrastrar una mayor duración en el tiempo previo (Rolland, 2004).

En las del segundo tipo, la enfermedad o discapacidad que tiene lugar tras una catástrofe o accidente, terminará integrándose dentro de una red de transacciones familiares problemáticas que existían con anterioridad a la ocurrencia de la mencionada catástrofe. En esta situación, si el profesional de la psicología se centra e implica excesivamente en la enfermedad o situación de discapacidad misma, puede que la familia también se agarre a ello sin querer entrar a trabajar y elaborar toda la problemática previa que es a la vez la base de su disfunción.

Así, el psicólogo pasaría a formar parte de un triángulo entre la familia y el paciente, triángulo que es comparable al que tienen lugar cuando los padres, ante la presencia de la enfermedad en la familia, se agarran a dicha patología para no hacer frente a los problemas maritales que se están dando en su unidad familiar (Minuchin, Rosman y Baker, 1978).

En este punto se hace radicalmente importante localizar cuándo la comunicación en una familia se encuentra bloqueada, ya que la psicosomática puede encontrar su campo de cultivo en estas familias, haciendo aflorar afecciones físicas que, en realidad ocurren por una problemática de origen psicológico, relacional y de comunicación entre los miembros, concretamente (Grifith y Griffith, 1994). Para este tipo de familias la expresión de un síntoma de tipo somático supone la sobrecarga del sistema familiar y pasa a funcionar como un moderador de toda situación que se ha vivido como problemática o peligrosa. Es una forma de desviar el foco del problema y la ansiedad, materializando las preocupaciones y disfunciones de tipo psicológico en afecciones físicas.

En definitiva, cuando una familia funciona de una manera no saludable previamente a la aparición de la enfermedad o la discapacidad, pueden encontrar en la enfermedad misma el camino de expresión de su malestar, agravando los síntomas, mostrando un curso desfavorable o un incumplimiento del tratamiento dado el alto nivel de reactividad de base que imperaba en dicho sistema; esto sin duda es un aspecto al que el profesional de la salud mental deberá atender con detenimiento y cautela. 


\section{LA REACCIÓN ANTE LA MUERTE EN FUNCIÓN DEL TIPO DE APEGO}

Bowlby (1980) ya definió la pérdida como una de las experiencias más dolorosas a las que una persona podía tener que hacer frente por la impotencia sentida, llegando a ser el único consuelo viable el retorno del ser querido perdido. Esta pérdida se concibe como desequilibrante, pues tenderá a desorganizar la vida del doliente, incluyendo el equilibrio del sistema de apego. De esta manera y como ya se ha señalado, el retorno a la situación previa a la muerte no sería posible, sino que la persona, lejos de volver a la anterior normalidad, deberá trabajar en su reajuste, en la adaptación a la nueva situación, pues se ha establecido que nuestra personalidad, tras un duelo, quedaría cambiada de manera definitiva, reflejando ésta la historia de nuestros duelos (Weiss,1993).

De esta manera, nos referimos al duelo que se desencadena tras perder a una figura de apego, la cual se trata de una persona específica y preferida frente a las demás, la cual es representada como una figura más fuerte, capaz y protectora, con quien se estaría unido a través del tiempo. Así, el duelo definido como normal por Bowlby (1980) pasaría por cuatro fases: la primera corresponde a la fase de aturdimiento o shock frente a la noticia de la pérdida, la cual es caracterizada por la incredulidad de la situación. A continuación, tendría lugar la fase de anhelo y búsqueda del ser querido, basada en las sensaciones de abandono y desprotección, dando paso ésta a la fase de desorganización y desesperación, cuando se toma conciencia plena de la irreversibilidad de la pérdida. Finalmente tendría lugar la fase de reorganización en la que el doliente debe tolerar las intensas emociones que percibe a la vez que puede ir viendo como sus fuerzas se ven aumentadas poco a poco.

Las personas que han contado con un apego de tipo seguro verán esta tarea facilitada por haber vivido una menor ambivalencia en el vínculo, por poder contar con mayores recuerdos favorables con el ser querido difunto al que estaban íntimamente vinculados. Esta presencia interna que la persona fallecida puede aportarle al doliente podría estar desempeñando diferentes papeles, desde un aporte de consuelo, compañía y energía protectora, hasta un conflicto ambivalente vivido con amenaza; esto es lo que estaría dando lugar a que un duelo fuera normal o fuese patológico.

El duelo es patológico cuando se cronifica, cuando éste es ausente o cuando se desencadena finalmente un episodio maniaco. Se trataría por tanto de la creencia acerca de que la pérdida es reversible, por lo que el proceso no se termina de completar y la persona no termina de reorganizar su vida tras 
el fallecimiento del ser querido; de esta forma, la persona no podría llegar a modificar sus modelos operativos internos ni las representaciones del self, las cuales hacen alusión en definitiva al apego adulto. Así, puede concluirse que son tres factores los que influyen en el proceso de duelo, los cuales se relacionan con el apego del doliente: el estilo de vínculo y apego que éste ha tenido durante su infancia, los patrones vinculares característicos de su vida adulta y la calidad y el tipo de vínculo que tenía con el ser querido fallecido. Analizando estos aspectos, se puede tener información de gran valor sobre el diagnóstico y el pronóstico del duelo al que deba hacer frente el doliente, dando directrices posibles en el trabajo psicoterapéutico a realizar con dicha persona (Montouri, 2013).

De esta manera, la explicación que Bowlby (1973) dio a la existencia de este proceso de duelo fue la ansiedad de separación que tenía lugar al vivir la pérdida de un vínculo, siendo el duelo la respuesta esperable y frecuente tras tener lugar dicha pérdida; es en las etapas iniciales del duelo, cuando se daría el trastorno emocional que tiene lugar en adultos, debido a la ruptura del vínculo. Fue el mismo Bowlby quien señaló una serie de factores que tendrían la capacidad de afectar el curso de este proceso de duelo, siendo estos: la identidad y el rol que tenía el fallecido, la edad y el sexo de la persona que estaba afrontando el proceso de duelo, las causas y circunstancias en las que había tenido lugar la pérdida, el contexto tanto social como psicológico del doliente y la personalidad característica del mismo, siendo éste último el que tenía mayor relevancia y peso en el proceso.

Cabe señalar que estilos de apego inseguro pueden dar lugar a lo que se ha denominado como Síndrome de Duelo Ambivalente, tratándose de una reacción ante la muerte caracterizada por una ambivalencia en la cual inicialmente puede aparecer el alivio para dar lugar más tarde a sentimientos también de dolor y desesperanza, los cuales harían su aparición con el paso del tiempo, dando lugar a un proceso intenso, duradero, con características auto-punitivas. Esto tendría lugar en relaciones caracterizadas por la complicación vincular, habiéndose podido producir incluso maltrato o abuso (Parkes y Weiss, 1983).

\section{INTERVENCIÓN TERAPÉUTICA SOBRE EL DUELO}

Para actuar sobre el duelo se han señalado diferentes niveles que son en realidad progresivos en complejidad, los cuales deben ir sucediéndose unos a otros: en primer término debe tener lugar la facilitación del ritual 
funerario, con el fin de poder despedir al difunto, facilitando el tránsito del cuerpo y el espíritu a un status distinto, ayudando así a los presentes a enfrentar su muerte ya que esto refleja la realidad de la pérdida y permite la expresión pública del dolor, pudiendo así llegar a comprobar que la vida y el grupo continúa, siendo esto una forma de celebrar la vida incluso (Thomas, 1985).

Posteriormente, podría tener lugar la intervención centrada en ofrecer consejo y apoyo, con los cuales se tiende a ayudar al doliente a completar sus asuntos inacabados con su ser querido, así como facilitando la despedida que pudo no haberse dado. Así, los objetivos se centrarían en aceptar la evidencia de que la persona no va a regresar, poder afrontar el dolor, poder reorganizarse nuevamente en una vida sin la persona fallecida, reubicando al ser querido para poder reanudar la propia vida (Worden, 1997).

Finalmente tendría lugar la terapia de duelo complicado propiamente dicha, la cual supone elaborar problemas complejos y profundos ocurridos dentro del sistema familiar, realizando dicho trabajo en un momento en el que la vida emocional está especialmente removida y sensibilizada. De esta manera se combinan el abordaje individual con el familiar ya que, si no se incluye a toda la familia, más allá del miembro identificado como el principal doliente, la desestructuración ocurrida por la pérdida puede terminar afectando a todos los miembros del sistema familiar (Pereira, 2002).

Las sesiones de terapia son intensas, con abundantes y útiles silencios, facilitando la expresión de las emociones y la posterior recuperación tras compartirlas. En este punto parece esencial hacer una redefinición positiva de dicha expresión emocional, incluidas la ira, la rabia o el miedo, especialmente en el caso de los hombres, a los que generalmente esto les supone una tarea de mayor dificultad. El empleo de rituales y técnicas movilizadoras psicodramáticas es de gran utilidad, tales como la silla vacía para poder traer al fallecido a sesión, el libro de memorias y recuerdos, el lenguaje más evocativo y narrativo, el empleo de cartas para poder comunicarle aspectos importantes al difunto, etc.

\section{CONCLUSIONES}

- Las demandas que la situación de pérdida suponen para un sistema familiar no son las mismas si la muerte tiene lugar de manera repentina o si esta se produce tras un periodo de enfermedad en el que sus miembros han podido prepararse para el proceso de duelo. 
- No hay una duración estimada o esperable para el proceso de duelo, consistiendo éste en un progreso sucesivo de etapas que deben ir viviéndose y superándose de forma exitosa.

- El acompañamiento psicológico y el trabajo psicoterapéutico se convierten así en aspectos fundamentales, tanto para poder desarrollar este difícil proceso de manera adecuada, posibilitando la expresión de emociones y sentimientos, como para la preparación para la muerte inminente de un ser querido, en el caso de etapas terminales de enfermedad.

- Tras una pérdida, todo sistema familiar debe reestructurarse, pues no se trata del mismo sistema, sino de uno nuevo que tendrá que volver a ponerse en funcionamiento sin el ser querido que ha fallecido.

- La reestructuración será diferente en función de quien sea el miembro de la unidad familiar que haya muerto, pues sus funciones y roles quedarán ausentes. Una ausencia en el subsistema parental produce la mayor desestructura posible, por la dependencia que los hijos tienen de sus progenitores.

- Existen diferencias en torno al género, siendo las mujeres quienes generalmente presentan duelos más intensos y duraderos, presentando sintomatología depresiva y ansiógena, en comparación con los hombres, que expresarían su sufrimiento a través de la ira, principalmente.

- En los casos en los que la muerte no es el desenlace final, aunque si uno posible en el futuro, habiendo quedado impedido en mayor o menor medida, la persona que queda encargada de su cuidado recibe el nombre de cuidador primario, recayendo sobre él una serie de responsabilidades y tareas que pueden suponer una carga tanto física como psicológica que deberán ser abordadas y trabajadas.

- El apoyo social, más allá del propio sistema familiar, que está viviendo el mismo proceso, se hace un aspecto de crucial importancia para poder desarrollar la tarea de manera exitosa. Se trata de un factor con funciones protectoras ante la pérdida o la enfermedad.

- Es necesario atender a los movimientos centrípetos y centrífugos que pueden suponer dentro del sistema familiar la pérdida de uno de sus miembros, observando así si se tiende a la cohesión o a la dispersión.

- Las creencias familiares sobre cómo debe ser el rol de enfermo o el rol de cuidador primario son también aspectos necesarios a tener en cuenta al situarnos frente a una persona que se encuentra en proceso de duelo, pues en el trabajo psicoterapéutico con ella deberá ser tenido en cuenta para poder ayudarles de manera personalizada, 
atendiendo así a sus necesidades. La adaptación es el punto clave a desarrollar por parte de un profesional.

- La importancia de los rituales es fundamental para poder desarrollar el proceso de duelo de manera exitosa. Son beneficiosos para las familias, pues ayudan a tomar conciencia de la pérdida, así como posibilitan la despedida del ser querido.

- El sistema de creencias familiar funciona como una guía ante la enfermedad, pudiendo ser un mediador o traductor entre la experiencia que uno mismo tiene acerca de la muerte o la enfermedad y la que tendría la familia.

- La actitud prudente y servicial del psicólogo en estos momentos parece un aspecto de gran relevancia para poder ser una fuente de ayuda a las familias que atraviesan momentos difíciles de perdida y duelo.

- Es importante atender a la posibilidad de la existencia de una disfunción previa en la unidad familiar, y aunque no vaya a abordarse en un primer momento, debe tenerse en cuenta para no encapsularse en el trabajo de duelo de manera exclusiva, obviando así un aspecto en la dinámica familiar previa que podría ser disfuncional.

- Bowlby habló de un proceso de duelo caracterizado de nuevo por un tránsito de etapas que irían reconduciendo a la persona a la aceptación del dolor y la pérdida del vínculo con la persona fallecida.

- Las personas con un apego seguro estarían mejor preparadas o predispuestas para realizar esta tarea de una manera más sencilla o exitosa, al no contar con reacciones ambivalentes ante el vínculo.

- El trabajo psicoterapéutico debe ir encaminado a la facilitación de la expresión emocional, a la elaboración de rituales y procesos vivenciales que ayuden a los familiares a conectar con su dolor y a favorecer el proceso de despedida.

\section{REFERENCIAS}

Bowlby, J. (1973). El Apego y la Pérdida, vol. II: Separación. Barcelona: Paidós.

Bowlby, J. (1980). Attachment and Loss, vol. III: Loss. London: The Hogarth Press.

Cobos, F., Espinoza, J., Portillo, J., y Rodríguez, G. (2002). La familia en la enfermedad terminal. Medicina de familia, 3(4), 44-50.

Dávalos, E. G. M., García, S., Gómez, A. T., Castillo, L., Suárez, S. S., y Silva, B. M. (2008). El proceso del duelo. Un mecanismo humano para el manejo de las pérdidas emocionales. Revista de Especialidades Médico-Quirúrgicas, 13(1), 28-31.

Davidhizar, R. (1992). Entendiendo la impotencia de los miembros de familia cuidadores de enfermos crónicos. Geriatric Nursing, 13(2), 66. 
Espina, A., Gago, J., y Pérez, M. (2005). Sobre la elaboración del duelo en terapia familiar. Revista de psicoterapia, 4(13), 77-87.

Feigin, R. (1994). Spousal adjustment to a postmarital disability in one partner. Family Systems Medicine, 12(3), 235.

Gil-Juliá, B., Bellver, A., y Ballester, R. (2008). Duelo: Evalauación, diagnóstico y tratamiento. Psicooncología, 5(1), 103-116.

Griffith, J., y Griffith, M. (1994). The Body Speaks: Therapeutic Dialogues for Mindbody Problems. Basic Books.

Grinberg, L. (1963). Sobre dos tipos de culpa: su relación con los aspectos normales y patológicos del duelo. Revista de psicoanálisis, 20(4), 321-332.

Kleinman, A. (1988). The illness narratives: Suffering, healing, and the human condition. Basic books.

Kluckhohn, F. R. (1958). Variations in the Basic Values of Family Systems. Social Casework, 39 (2-3), 63-7.

Millán-González, R., y Solano-Medina, N. (2010). Duelo, duelo patológico y terapia interpersonal. Revista colombiana de psiquiatría, 39(2), 375-388.

Minuchin, S., Rosman, B. L., y Baker, L. (1978). The anorectic family. Psychosomatic families: Anorexia nervosa in context, 51-73.

Montuori, E. (2013). El duelo visto desde la teoría del apego. Recuperado de: http:// apra. org. ar/pdf/mayo/montouri. pdf.

Parkes, C.M. \& Weiss, R. (1983). Recovery for bereavement. New York. Basic Books.

Pereira, R. (2002). Duelo: desde el punto de vista individual al familiar. Sistemas Familiares 18(1-2), 48-61.

Pérez-Cárdenas, C., y Sebazco-Pernas, A. (2000). Familia perdida: Características de esta crisis familiar. Revista Cubana de Medicina General Integral, 16(1), 93-97.

Roitman, A., Armus, M., y Swarc, N. (2002). El duelo por la muerte de un hijo. Aperturas psicoanalíticas: revista internacional de psicoanálisis, 12.

Rolland (2000). Familias, enfermedad y discapacidad, una propuesta desde la terapia sistémica. Gedisa.

Sontag, M. S. \& Schlater, J. D. (1982). Proximity of clothing to self: Evolution of a concept. Clothing and Textiles Research Journal, 1(1), 1-8.

Tercero, R. P., y Navarra, D. D. (2010). Evolución y diagnóstico del duelo normal y patológico. $F M C, 17(10)$, 656-63.

Thomas, L. V. (1985). Rites de mort. Pour la puix des vivants. París: Fayard.

Toro, L. B., Flores, S. L., Gil, J. R., Domínguez, R. C., Marín, T. G., y Prado, M. A. (2007). Perfil del cuidador principal del enfermo en situación terminal y análisis del riesgo de desarrollar duelo patológico. Medicina paliativa, 14(3), 0.

Volker, T., Striegel, P., Dudley, D., Wilkins, J., y Gibson, D. (1997). Parental grief of a perinatal loss: A comparison of individual and relationship variables. Journal of Personal and Interpersonal Loss: International Perspectives on Stress \& Coping, 2, 167-187.

Walsh, F. (1993). Conceptualization of normal family processes. New York, Guilford.

Weiss, R. (1993) «Loss and recovery» en: Stroebe, M., Stroebe W., y Hanson, R. O.

(Eds.), "Handbook of bereavement: Theory, research an intervention». Cambridge: University Press.

Wolin, S. J., y Bennett, L. A. (1984). Family rituals. Family process, 23(3), 401-420. 
Worden, J. (1997). El tratamiento del duelo: asesoramiento psicológico y terapia. Barcelona: Paidós.

Yoffe, L. (2003). El duelo por la muerte de un ser querido: creencias culturales y espirituales. Psicodebate, 3, 127-158.

Zborowski, M. (1969). People in pain. San Francisco: Jossey-Bass. 